\title{
Reconfigurable measuring system for the automatic detection of bacterial growth in a specimen processing platform
}

\author{
Paolo Bellitti ${ }^{1}$, Michele Bona ${ }^{1}$, Michela Borghetti ${ }^{1}$, Emilio Sardini ${ }^{1}$, Mauro Serpelloni ${ }^{1}$, Stefania \\ Fontana ${ }^{2}$
}

1 Università degli Studi di Brescia, Via Branze 38, 25123 Brescia, Italy

2 COPAN Italia S.p.A., Via F. Perotti 10, 25125 Brescia, Italy

\begin{abstract}
The detection of bacterial infections in biological samples represents a fundamental task in human health preservation. This paper describes a measuring system that aims to detect bacterial growth in Petri dishes while they are inside the incubator of the WASPLab® specimen processing platform (COPAN Italia S.p.A.). In fact, the interaction between the system and the WASPLab ${ }^{\circledR}$ enhances the analytical process. The proposed system monitors bacterial growth by measuring the impedance related to the examined Petri dishes. Furthermore, it can be easily reconfigured according to user requirements. Finally, it has some features that enhance its connectivity with the WASPLab ${ }^{\circledR}$ and other devices, developing a structure that is compliant with the Industry 4.0 model. We assess the system performance through different tests, in which we monitored the growth of $S$. aureus ATCC 6538 , starting from concentrations in the order of $4.5 \cdot 10^{8} \mathrm{CFU} / \mathrm{ml}, 4.5 \cdot 10^{7} \mathrm{CFU} / \mathrm{ml}$, and $4.5 \cdot 10^{6} \mathrm{CFU} / \mathrm{ml}$. The results show the system's correct operation both in an ad hoc setup and while working with the WASPLab ${ }^{\circledR}$. Furthermore, the connectivity between the system and other devices allows for a user to be informed about the analyses that are on course and to act on the system at any time and even from remote locations.
\end{abstract}

\section{Section: RESEARCH PAPER}

Keywords: bacterial growth detection; WASPLab® platform; impedance; Petri dish; Industry 4.0

Citation: Paolo Bellitti, Michele Bona, Michela Borghetti, Emilio Sardini, Mauro Serpelloni, Stefania Fontana, Reconfigurable measuring system for the automatic detection of bacterial growth in a specimen processing platform, Acta IMEKO, vol. 8, no. 2, article 6, June 2019, identifier: IMEKO-ACTA-08 (2019)02-06

Section Editor: Alessandro Depari, University of Brescia, Italy

Received July 20, 2018; In final form June 07, 2019; Published June 2019

Copyright: This is an open-access article distributed under the terms of the Creative Commons Attribution 3.0 License, which permits unrestricted use, distribution, and reproduction in any medium, provided the original author and source are credited.

Funding: This work was supported by the Italian Ministry for Education, University and Research, as part of the Adaptive Manufacturing project (CTN01_00163_216730), which was promoted by the Italian 'Cluster Tecnologico Nazionale Fabbrica Intelligente'.

Corresponding author: Michele Bona,email: m.bona002@unibs.it

\section{INTRODUCTION}

Worldwide bacterial infections are a major concern for human health according to reports and recommendations from the World Health Organization [1]-[2]. Emerging pathogens, together with antimicrobial resistances spreading within bacteria species, challenge microbiology laboratories to achieve rapid and efficient detection of bacterial presence in biological samples.

In the past few decades, research activities in academia and industry have led to the development of techniques permitting the detection of bacterial growth in a biological sample much earlier than by traditional colony count methods [3]-[4]. In this way, positive specimens are recognised faster and can be further analysed, allowing the eventual provision of appropriate therapy for patients. Such techniques include fluorescence spectroscopy [5], impedance microbiology [6], surface plasmon resonance [7], isothermal microcalorimetry [8], and flow cytometry [9]. At the same time, increasing laboratory automation enables the automated management of specimen handling, plating, and incubation, reducing the processing time and improving the quality of results [10]. The combination of such techniques with automation has resulted in the appearance on the market of systems aiming to meet the abovementioned goals. For instance, both the BACTEC ${ }^{\mathrm{TM}}$ (Becton, Dickinson, and Company) [11][12] and the BacT/ALERT (bioMerieux SA) [12]-[14], in their different versions, monitor the change in fluorescence caused by 


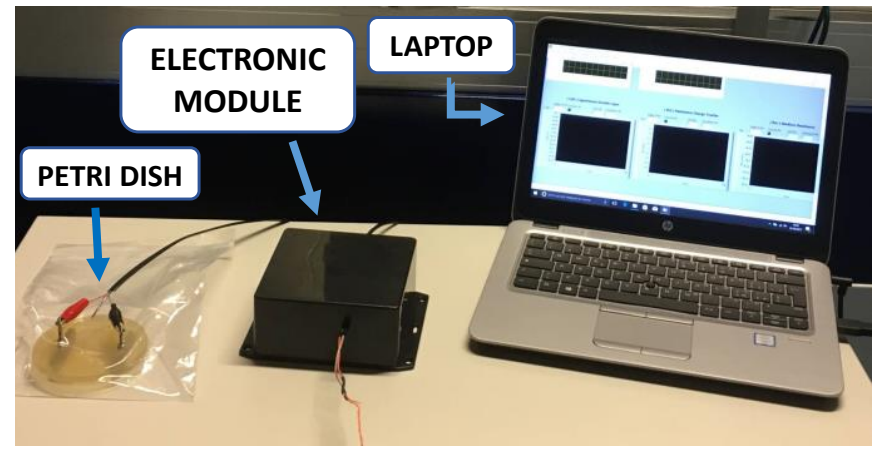

Figure 1. Picture of the developed measuring system.

a $\mathrm{CO}_{2}$ increase as a by-product of bacterial activity. The RABIT (Don Whitley Scientific Ltd.) [15] and the BacTrac (SY-LAB Geräte Gmbh) [16] measure electric impedance, whose variation is related to pathogen growth. The BIAcore system (GE Healthcare) monitors an optical signal that is dependent on bacterial presence [17]. The Thermal Activity Monitor (TA Instruments) detects heat produced by the chemical reactions associated with the growth [8].

Within automated solutions, the Walk-Away Specimen Processor ${ }^{\mathrm{TM}}$ WASP ${ }^{\circledR}$ from COPAN Italia S.p.A. is a benchmark for the automatic and efficient elaboration of clinical samples, improving the quality of microbiological analyses and laboratory reports [18]. The WASP ${ }^{\circledR}$ is integrated in a more complex specimen processing platform called WASPLab® [19]. This platform combines the WASP ${ }$ with $\mathrm{CO}_{2}$ and non- $\mathrm{CO}_{2}$ incubators, an image acquisition station for each incubator, and a digital imaging interface. It monitors bacterial growth by taking images of incubated Petri dishes, which contain biological samples for analysis. In this way, a considerable number of highquality plate images is produced, with positive repercussions also for species identification and antibiotic susceptibility tests [20].

As well as the most advanced manufacturing systems, the WASPLab® is intended to achieve higher and higher throughput quality standards. In addition, a market that is in constant evolution requires systems to adapt promptly to its increasingly fast dynamics. One example is the increasing number of requests for system and/or product customisation and reconfiguration to meet end-user requirements [21]. This is especially true in the Industry 4.0 age, when systems are conceived to be connected with other devices that work in cooperation and create a Smart Factory scenario [22]-[23]. With reference to the WASPLab®, the precocious detection of bacterial growth on incubated plates, besides and independent from image acquisition, could be an unprecedented feature that may further enhance its analytical performance.

Among the abovementioned techniques, impedance microbiology represents one of the most suitable ways of achieving this goal. In fact, impedance measurement allows for the acquisition of quantitative data about bacterial growth through relatively simple and cheap instrumentation [4]. In a previous work [24], we illustrated some results from a study in which we applied an impedance measuring system to analyse bacterial culture plates inside one of the WASPLab® incubators. In this article, we present such system, and we describe the laboratory tests performed to evaluate the possibility of its integration in the WASPLab® in more detail. In fact, the system could ensure continuous growth monitoring, bringing a great advantage to precocious detection: The opportunity to interrupt incubation could be individually determined for each single plate,

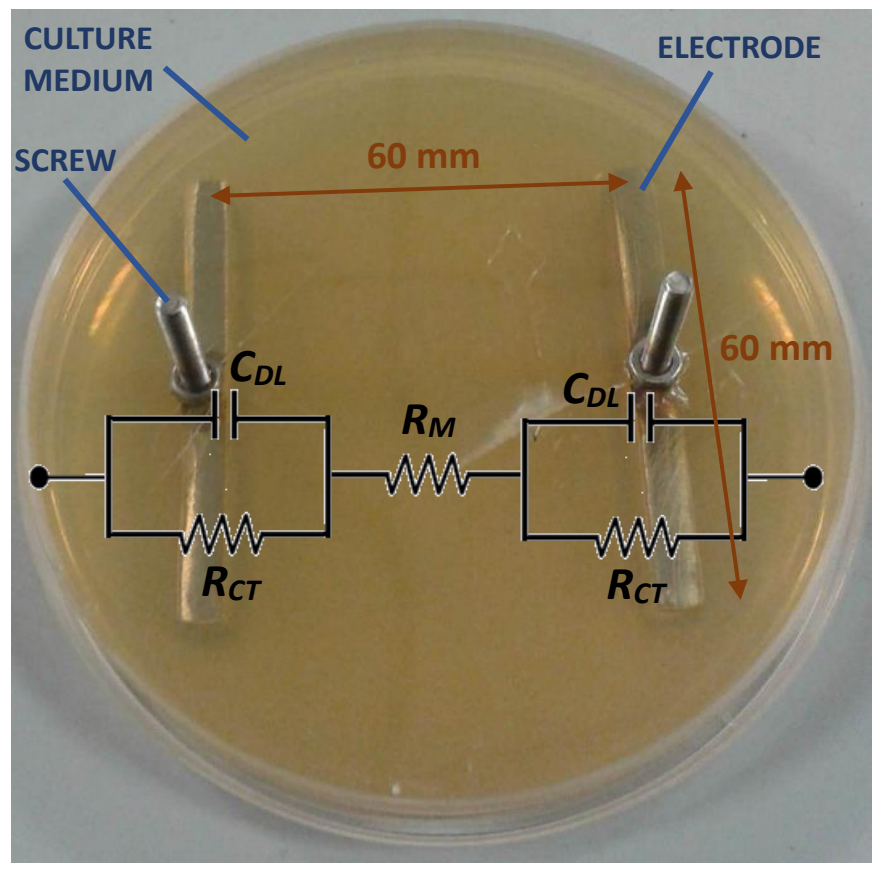

Figure 2. Instrumented Petri dish with its equivalent model.

avoiding pre-selection at specific time intervals. Further analysis, like plate image acquisition, colony-picking, species identification, and antibiotic resistance determinations, could be performed promptly and without wasting time. In addition, this article is different with respect to the works presented in the literature review. While such works deal specifically with sensor design and related growth detection techniques, this article describes a complete system with a sensor, electronics, and a user interface. Then, it focuses on system characteristics, combining impedance measurement capability with high flexibility and reconfigurability. Finally, it stresses the system features that enhance collaboration and/or integration with other platforms (e.g. the WASPLab®) and connectivity with external devices. Therefore, it presents a case study about the application of Industry 4.0 principles to microbiology. At the same time, this article proposes an improved system compared with previous versions illustrated in other works [25]-[26], regarding both electronics and user interface development.

The article is structured as follows. In Section 2, we provide a detailed description of the measuring system, also highlighting the progress accomplished with respect to its previous versions. Then, in Section 3, we illustrate how we carried out the tests on the system, whose results are reported in Section 4. Finally, the work's achievements are summarised and concluding remarks are given.

\section{THE DEVELOPED MEASURING SYSTEM}

Figure 1 provides a picture of the measuring system. Three main parts can be identified. The first part is a Petri dish instrumented with a pair of electrodes. The second part is an electronic module designed to measure Petri dish impedance. Finally, the third one is a laptop running a user interface program.

\subsection{Petri dish}

Figure 2 shows an employed Petri dish which was realised by inserting two stainless steel electrodes in a plate of the same type as those analysed with the WASPLab®. The inserted electrodes 
are $60 \mathrm{~mm}$ long and have a rectangular section. They are partially immersed in the agar culture medium contained in the Petri dish, at a height of about $4 \mathrm{~mm}$. In addition, they are kept positioned at a distance of $60 \mathrm{~mm}$ in order to allow the generated electric field to cover the greatest possible medium surface. Finally, they are fixed to the plate cover, from which two steel screws protrude as contact terminals. All Petri dishes were sterilised with $\gamma$ rays after the insertion of electrodes.

We fitted the Petri dish frequency response with the equivalent circuit model represented in Figure 2 [25]. It describes the electrode/medium interface through the parallel between the double layer capacitance $C_{\mathrm{DL}}$ and charge transfer resistance $R_{\mathrm{CT}}$. Then, the resistance $R_{M}$ defines the medium's own conductance. It is derived from the Randles circuit [27], without Warburg impedance, whose contribution can be neglected in the frequency range analysed in our work, going from some tens of hertz to less than $1 \mathrm{kHz}$. In this model, bacterial growth is associated with a change in the characteristics of both medium and electrode/medium interface, which is reflected by $R_{M}, C_{D L}$, and $R_{C T}$ variations. Such parameters are calculated from Petri dish impedance measurements carried out at two constant frequencies $f_{1}$ and $f_{2}$, by implementing the following mathematical formulae:

$$
\begin{aligned}
& C_{D L}=\frac{f_{1} / \operatorname{Im}\left(Z\left(f_{1}\right)\right)-f_{2} / \operatorname{Im}\left(Z\left(f_{2}\right)\right)}{\pi f_{2}^{2}-\pi f_{1}^{2}} \\
& R_{C T}=\sqrt{-\frac{\operatorname{Im}\left(Z\left(f_{1}\right)\right)}{4 \pi f_{1} C_{D L}+4 \pi^{2} f_{1}^{2} C_{D L}^{2} \operatorname{Im}\left(Z\left(f_{1}\right)\right)}} \\
& R_{M}=\operatorname{Re}\left(Z\left(f_{2}\right)\right)-\frac{2 R_{C T}}{1+4 \pi^{2} f_{2}^{2} C_{D L}^{2} R_{C T}^{2}}
\end{aligned}
$$

where $\operatorname{Im}\left(Z\left(f_{1}\right)\right)$ and $\operatorname{Im}\left(Z\left(f_{2}\right)\right)$ are the values of the impedance imaginary part measured at $f_{1}$ and $f_{2}$, respectively, whereas $\operatorname{Re}\left(Z\left(f_{2}\right)\right)$ is the value of the impedance real part at $f_{2}$. The need to always measure $\operatorname{Im}(Z)$ and $\operatorname{Re}(Z)$ at two frequencies allows for working with a system characterised by simpler electronics.

\subsection{Electronic module}

Figure 3 reports the block scheme of system electronic module, which excites Petri dish electrodes with sinusoidal waveforms and allows measuring related impedance $Z$. Such module was assembled from off-the-shelf components.

The main component is the AD5933 integrated circuit (Analog Devices, Inc.) [28]. It integrates elements that otherwise should be included as separate parts. An example is the direct digital synthesiser for excitation waveforms generation, which was a distinct element in the previous electronic unit of system version proposed in [25]. It is a part of the same component in the present version. This results in benefits concerning reduced dimensions, which enhance system portability. In fact, the electronic module is contained in the black box shown in Figure 1 , whose dimensions are $120 \times 120 \times 55 \mathrm{~mm}$, and it is more compact than the electronic unit described in [25]. Integrated circuit AD5933 generates excitation waveforms characterised by a DC offset equal to $1.65 \mathrm{~V}$, but whose amplitude and frequency can be set according to user necessity, albeit within a specific range, comprising $f_{1}$ and $f_{2}$. This gives a certain degree of flexibility to the entire system that is able to fit different operating conditions. Furthermore, AD5933 performs $Z$ measurements and makes the results available in terms of impedance real part and impedance imaginary part.

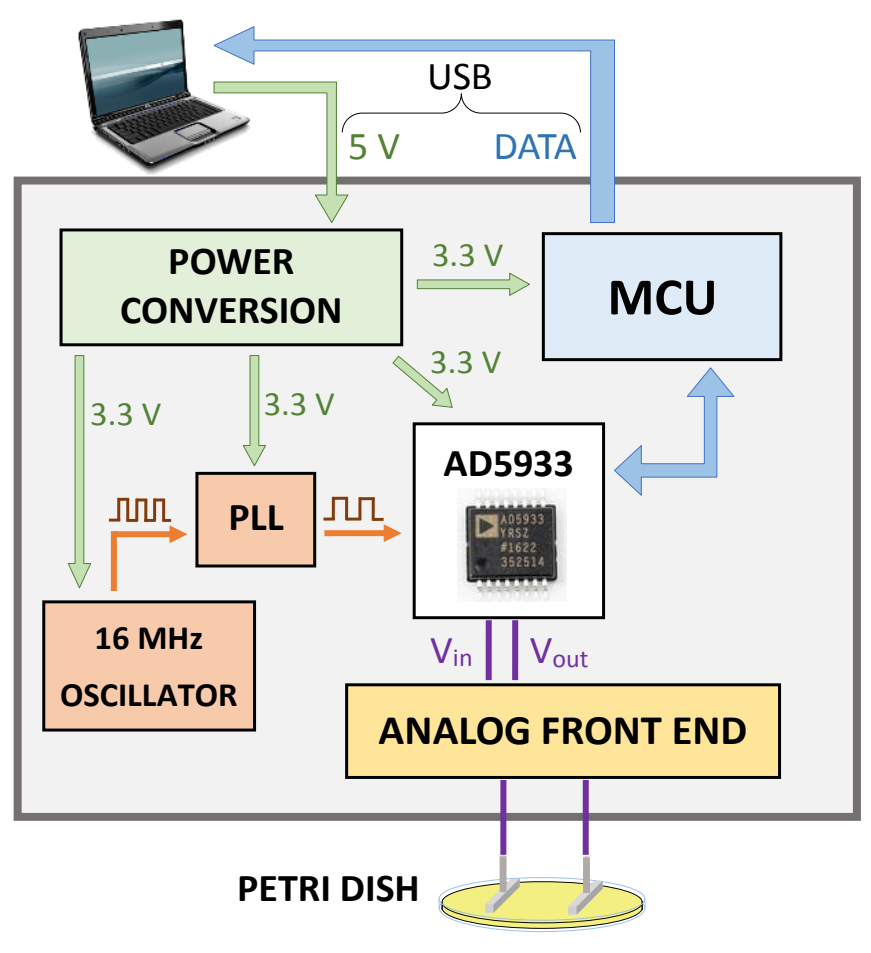

Figure 3. Block scheme of the system electronic module.

Other components guarantee electronic module operation (Figure 3). Firstly, the power conversion unit makes a $3.3 \mathrm{~V}$ proper supply available to all electronic circuits, from the $5 \mathrm{~V}$ supply provided by the laptop, which is connected by a USB port. Then, the AEL 4303 (AEL Crystals LTD) $16 \mathrm{MHz}$ oscillator in combination with the ADF4001 (Analog Devices, Inc.) phaselocked loop (PLL) divider guarantee the AD5933 clock frequency that is required according to the $f_{1}$ and $f_{2}$ range i.e. 200 $\mathrm{kHz}$ [28]. Anyway, other values of clock frequency can be achieved by changing the PLL division ratio (currently equal to 80 ), enabling reconfiguration of the measuring system (if necessary). Furthermore, a modifiable analog frontend was inserted between the AD5933 output and the instrumented Petri dish to filter and amplify the signals. Finally, the microcontroller (MCU in Figure 3) acts as an interface between AD5933 and laptop for data transmission, and it manages global module operation.

Before starting a new measurement session, the electronic module needs to be tuned. This step is necessary for finding the value of its internal gain factor and for compensating any parasitic effect due to the presence of cables. Tuning is carried out by connecting discrete elements (e.g. a resistor, a capacitor, or a combination thereof) of known value to module terminals, instead of a Petri dish.

Since the electronic module comprises commercial components, more identical samples can be replicated and activated simultaneously if there is a need to work on an increasing number of Petri dishes at the same time. This functionality favours the enhancement of measuring system modularity.

\subsection{Laptop}

The laptop runs a LabVIEW Virtual Instrument (VI) user interface program, which implements multiple functionalities. Although we used a laptop in this work to augment system portability, any computer that is able to run the VI can be 
potentially employed for the proposed measuring system. This VI is a more advanced version than the one previous characterising system prototype illustrated in [26]. In particular, we added some features that increase measuring system connectivity with collaborating devices, following an architecture that is compliant with Industry 4.0 paradigms. In general, the VI allows for:

- Setting the values of the measurement parameters, such as the working frequencies $f_{1}$ and $f_{2}$ and the amplitude of excitation waveforms. In addition, the user can choose to put the system in continuous operation by setting the measurement cycle duration and the number of cycles that will be performed. From that moment, the system is able to work autonomously until the last cycle terminates. The opportunity to select the values for different parameters makes the system very flexible, as it can be reconfigured to fit a particular need.

- Calculating the value of the electronic module gain factor.

- Driving the electronic module operation in such a way that system can perform automatic $Z$ measurements at frequencies $f_{1}$ and $f_{2}$.

- Elaborating on the measurement data in order to obtain the values of model parameters $C_{\mathrm{DL}}, R_{\mathrm{CT}}$, and $R_{\mathrm{M}}$ by implementing Equation (1), Equation (2), and Equation (3), respectively.

- Showing real-time data on the $Z$ and model parameters. Data is reported by using either graphical tools or numerical indicators. Therefore, the user can observe their variations at any moment during the entire measurement session.

- Evaluating $C_{\mathrm{DL}}, \mathrm{R}_{\mathrm{CT}}$, and $\mathrm{R}_{\mathrm{M}}$ trends in order to detect bacterial growth in an automatic way. In fact, at the beginning of the test, the user must set a threshold for the variation of each model parameter with respect to a reference value. When variations are relevant so as to exceed the thresholds for all parameters at the same time, an alarm is generated to inform the user that system has detected bacterial growth. Furthermore, once the alarm occurs, the VI creates a notification email automatically and sends it to a previously defined address by means of an SMTP server (only in the present VI version).

- Saving all data in *.txt files at the end of every cycle. Such files are contained in a specific folder on the laptop, which is shared on the cloud (only in the present VI version). In this way, they are available for elaboration and visualisation just after they are saved, not only on the laptop, but also from any permitted device with an Internet connection (including other PCs, smartphones, and tablets).

In addition, in this version, the laptop is accessible for remote monitoring and control at any time in the measurement session from external connected devices that have permission to access it. Therefore, even a user who is not in the laboratory can observe parameter trends in real time and be informed about bacterial growth events.

\subsection{Connectivity}

Compared to the previous versions illustrated in [25]-[26] in which connectivity was not an addressed issue, the solutions implemented by the measuring system proposed in this paper promote information exchange between the actors that play a role in the analytical process, as presented in Figure 4. This exchange is beneficial.

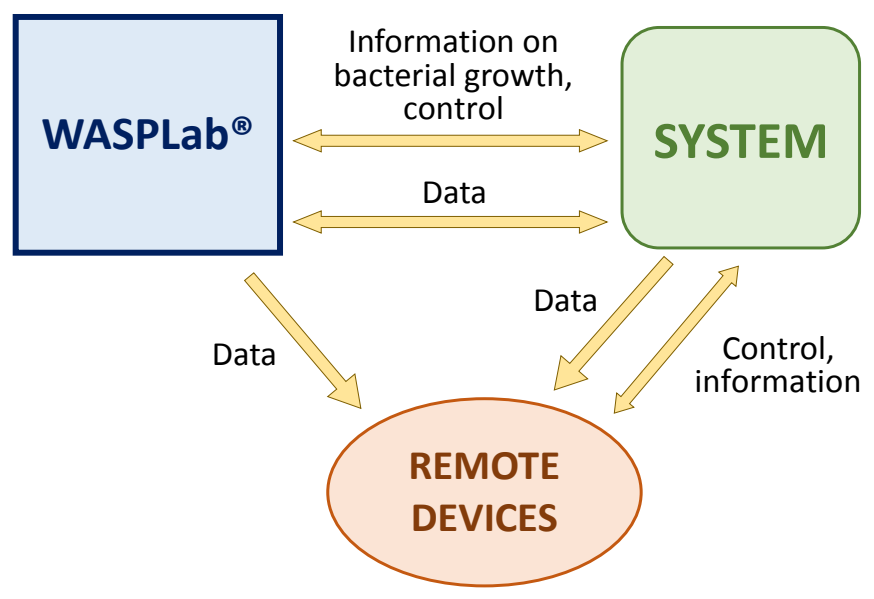

Figure 4. Communication between connected systems.

On the one hand, they allow collaboration between the

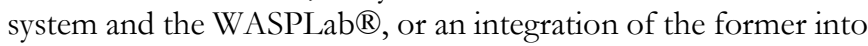
the latter, as illustrated in [24]. In fact, the WASPLab® can give a notification that one or more Petri dishes have been positioned inside an incubator for analysis. Then, the measuring system monitors the bacterial growth in those plates. Finally, the system alerts the WASPLab ${ }^{\circledR}$ about growth detection, and positive Petri dishes are analysed through image acquisition, or they are simply removed from the incubator for further examination. Such communication between them helps the development of a structure that is typical of the Industry 4.0 model and its application to the microbiology field.

On the other hand, data access that is granted to external connected devices permits selected personnel (e.g. engineers and microbiologists) to have information about bacterial growth at their disposal at any time and to be alerted once growth is detected, regardless of their location when the analysis is taking place. In addition, they will know if a problem is occurring, and they can exert remote control on system operation; for instance, they may start or stop data acquisition according to a particular need.

\section{THE PERFORMED TESTS}

We tested the impedance measurement performance of the electronic module. We found an accuracy of $0.8 \%$ by comparing its response with the values measured by a reference instrument (i.e. the HP4194A impedance analyser) for the same device under test [26]. This is comparable with the value provided by the AD5933 datasheet [28]. In addition, the repeated measurements on fixed components permitted the estimation of a standard deviation of $|Z|$ in the order of $10 \mathrm{~m} \Omega$.

In this paper, we focus on system capability in bacterial growth detection, which was evaluated through different tests.

\subsection{System preparation}

For every test, three instrumented Petri dishes were filled with a Tryptone Soy Agar (TSA) culture medium. We inoculated two plates with a solution containing equivalent values of an initial concentration $C_{0}$ of $S$. aureus ATCC 6538. Indeed, we took into account different orders of magnitude for $C_{0}$ in different tests i.e. 4.5.10 $\mathrm{CFU} / \mathrm{ml}, 4.5 \cdot 10^{7} \mathrm{CFU} / \mathrm{ml}$, and $4.5 \cdot 10^{6} \mathrm{CFU} / \mathrm{ml}$. Inoculation took place in a biological safety cabinet at ambient temperature. We did not inoculate the third plate as a control. 


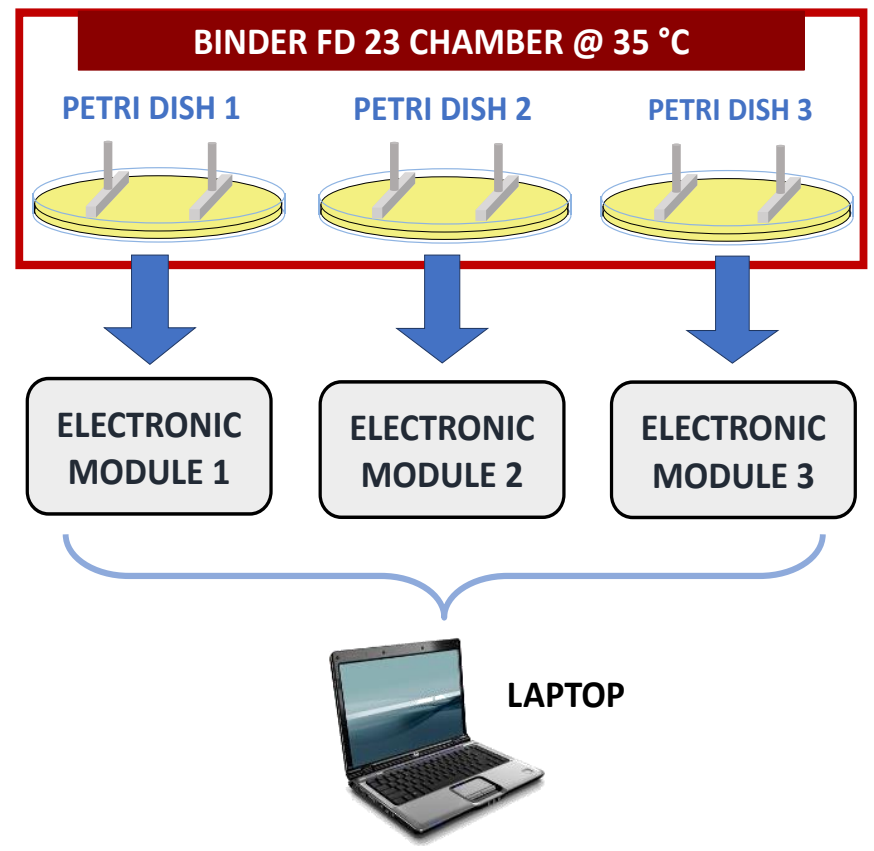

Figure 5 . Scheme of the setup realised for preliminary growth tests.

We employed three electronic modules, one for each Petri dish. In this way, we were able to evaluate the system operation when gathering data about more samples at the same time. Through the VI user interface, we set the amplitude of the provided excitation waveforms to $1 \mathrm{~V}_{\mathrm{pp}}$, while $f_{1}$ and $f_{2}$ were set as equal to $50 \mathrm{~Hz}$ and $150 \mathrm{~Hz}$, respectively. Then, we found the internal gain factor of each module by connecting a $100 \Omega$ commercial resistor to its terminals and evaluating the corresponding impedance output.

Finally, the VI was programmed for automatic bacterial growth detection based on the obtained values of $C_{\mathrm{DL}}, R_{\mathrm{CT}}$, and $R_{\mathrm{M}}$ related to each Petri dish. In particular, the working cycle duration was set to two minutes, while the number of cycles was defined so as to drive the system to work autonomously for 24 hours.

\subsection{Preliminary tests}

Firstly, preliminary tests were performed with an ad hoc laboratory setup in order to evaluate system operation (specifically in terms of autonomous growth monitoring over a day) under given conditions recreating the WASPLab ${ }^{\circledR}$ environment. Figure 5 shows setup block scheme.

Once the steps described in Section 3.1 were completed, we placed all Petri dishes in a FD 23 thermostatic chamber (BINDER GmbH). This chamber was exploited to simulate an incubator guaranteeing the environmental conditions (in terms of temperature and air ventilation) necessary for bacterial growth. It was turned on one hour before starting every test in order to achieve uniform conditions inside it. In addition, its internal temperature was set to $35^{\circ} \mathrm{C}$. Afterwards, we connected each Petri dish to a single electronic module. Finally, we started automatic data acquisition.

\subsection{Tests with the WASPLab ${ }^{\circledR}$}

Then, we conducted a series of tests in COPAN Italia S.p.A., aiming to monitor bacterial growth while the instrumented Petri dishes were contained in one of the WASPLab ${ }^{\circledR}$ incubators. Figure 6 shows the measuring system together with the WASPLab® during one of these tests.

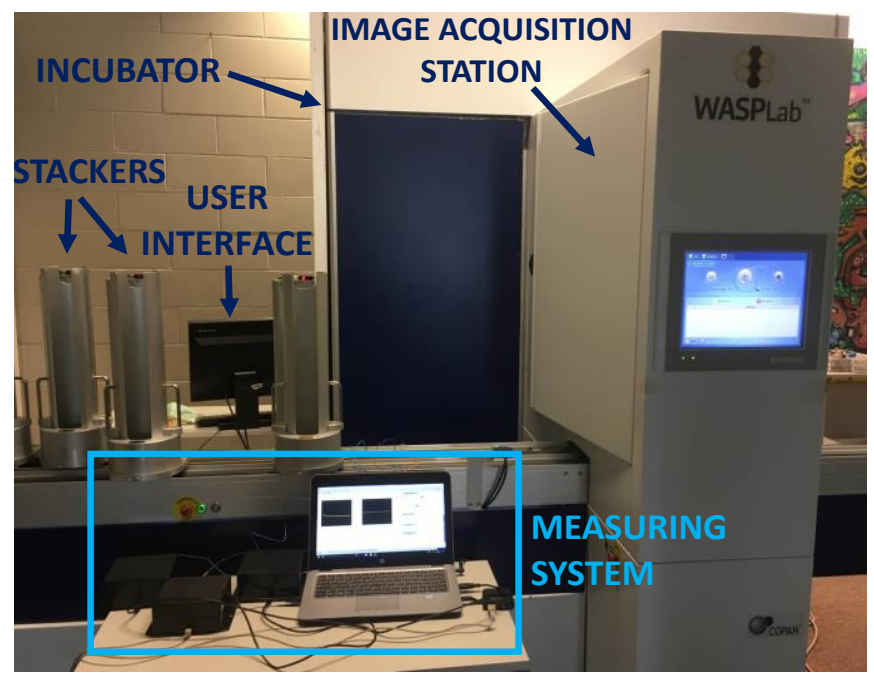

Figure 6. Measuring system with the WASPLab ${ }^{\circledR}$ platform during one of the tests conducted in COPAN Italia S.p.A.

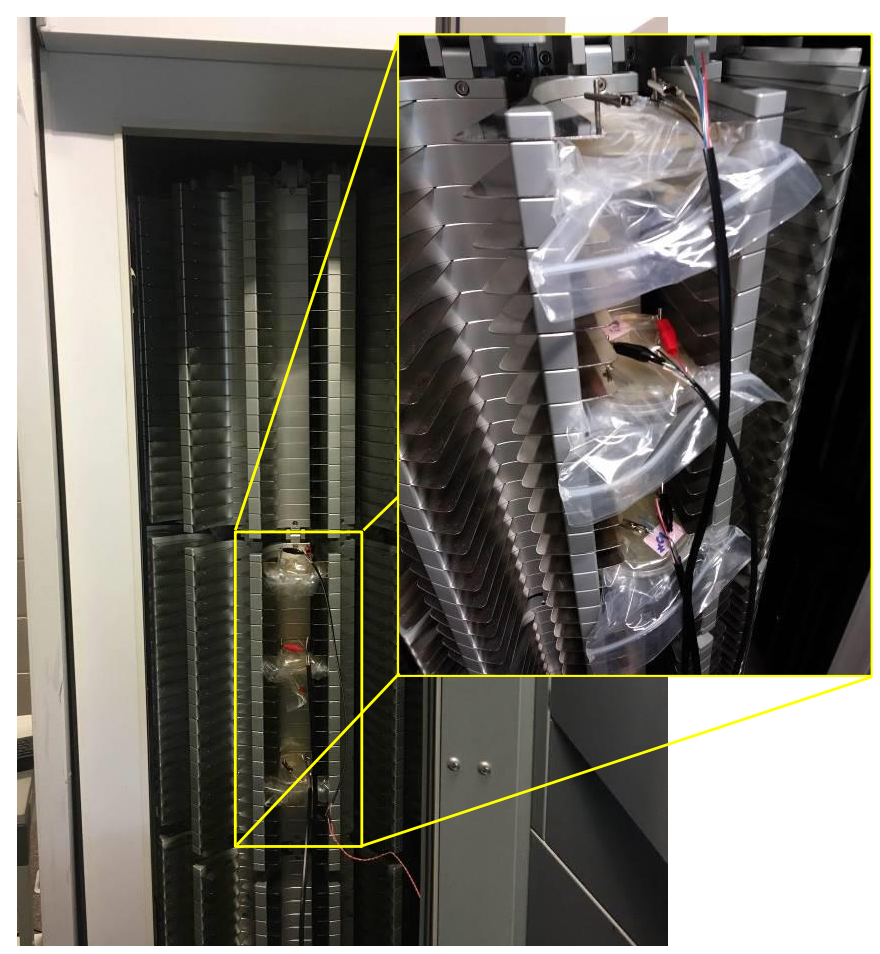

Figure 7. Petri dishes stacked in one of the WASPLab ${ }^{\circledR}$ incubators.

After the system was prepared (see Section 3.1), we put the Petri dishes in the incubator, as highlighted in Figure 7. As evidenced therein, there is a rack that allows for easy positioning of the Petri dishes, and it prevents them from moving during the test. The incubator had been already working when the Petri dishes entered, assuring uniform internal conditions. In particular, its control system maintained its internal temperature at $35 \pm 2{ }^{\circ} \mathrm{C}$ despite the occurrence of disturbances e.g. when opening the incubator at the beginning of the test to put the plates inside. Again, we connected the stacked Petri dishes to the electronic modules, and data acquisition began. 


\section{(4)}
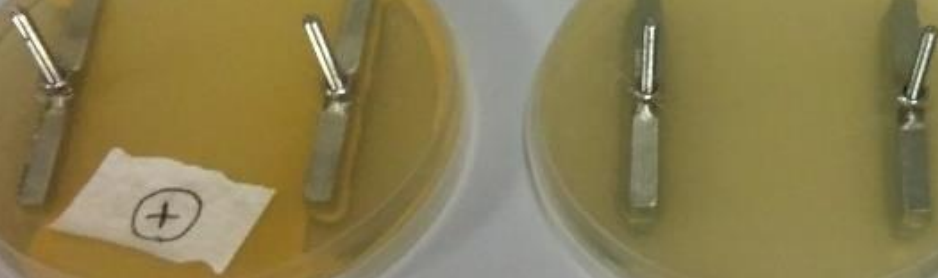

Figure 8. Petri dishes at the end of a preliminary test in which $C_{0}=4.5 \cdot 10^{8} \mathrm{CFU} / \mathrm{ml}$.

\section{RESULTS}

\subsection{Preliminary tests}

Figure 8 shows the Petri dishes that were analysed during one of the preliminary tests (in this case, $C_{0}=4.5 \cdot 10^{8} \mathrm{CFU} / \mathrm{ml}$ ). The inoculated plates are identified by the ' + ' label. The picture was taken at the end of the test, when the inoculated plates with bacterial growth can be easily distinguished from the noninoculated one (in the centre) according to a visual inspection of the plate. In addition, the bacterial growth produced a colour
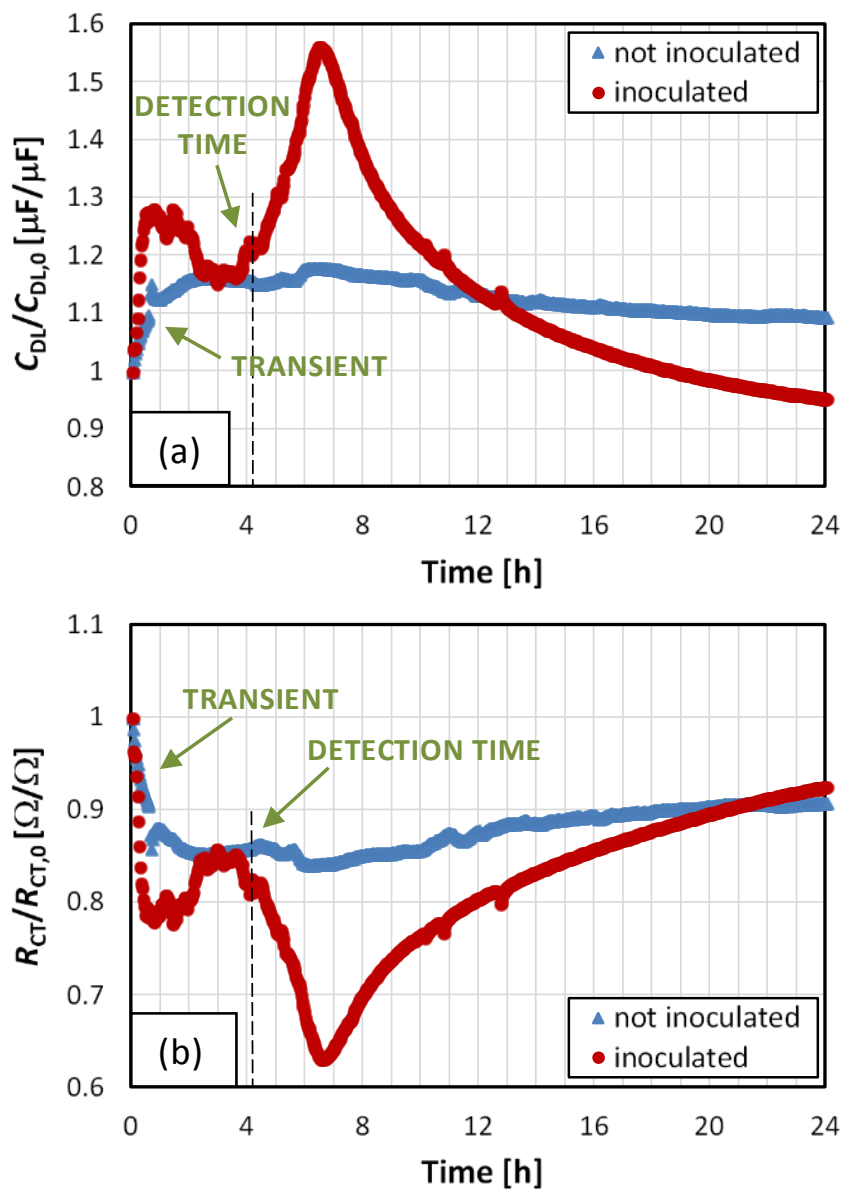

Figure 9. Time trend of electrode/medium interface parameters, resulting from one of the tests in which $C_{0}=4.5 \cdot 10^{8} \mathrm{CFU} / \mathrm{ml}$. Values are normalised with respect to those found at time $=0 \mathrm{~h}$ : (a) $C_{\mathrm{DL}} / C_{\mathrm{DL}, 0}$; (b) $R_{\mathrm{CT}} / R_{\mathrm{CT}, 0}$.
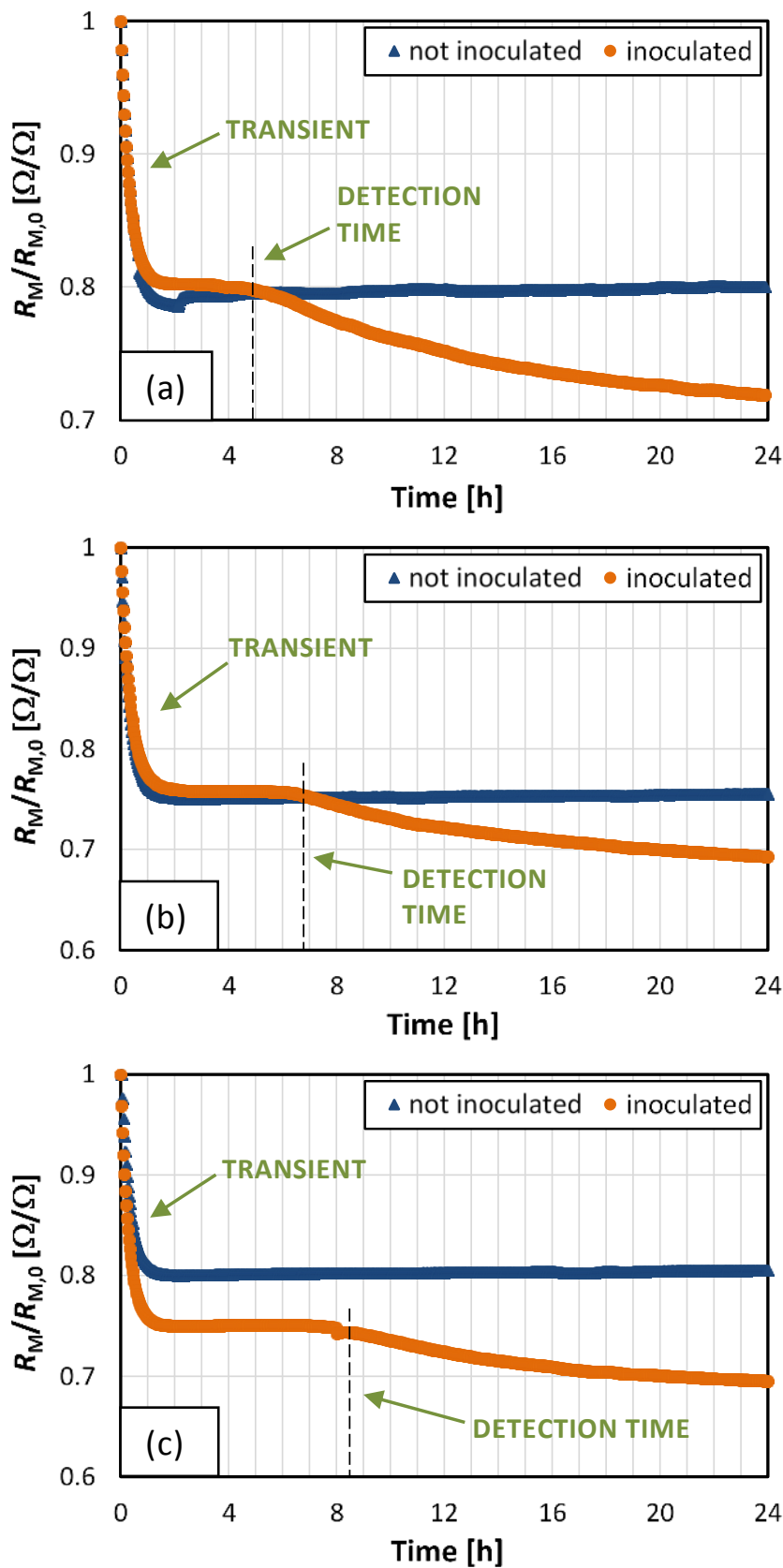

Figure 10. Time trend of resistance $R_{\mathrm{M}}$ normalised with respect to its value $R_{\mathrm{M}, 0}$ at time $=0 \mathrm{~h}$, resulting from a test in which (a) $C_{0}=4.5 \cdot 10^{8} \mathrm{CFU} / \mathrm{ml}$; (b) $C_{0}$ $=4.5 \cdot 10^{7} \mathrm{CFU} / \mathrm{ml}$; (c) $C_{0}=4.5 \cdot 10^{6} \mathrm{CFU} / \mathrm{ml}$. 
change in TSA culture medium, in correspondence of the inoculated surface, due to microbial presence. This discrimination between 'growth' and 'no growth' conditions, according to inoculated/not inoculated plate preparation, was sufficient for the purpose of this article's specific activity.

Figure 9 reports the time trend that characterises ing $C_{\mathrm{DL}}$ (Figure $9 \mathrm{a}$ ) and $\mathrm{R}_{\mathrm{CT}}$ (Figure $9 \mathrm{~b}$ ) for a Petri dish inoculated with $4.5 \cdot 10^{8} \mathrm{CFU} / \mathrm{ml}$ and a non-inoculated plate. In both cases, the values are normalised with respect to those found at time $=0 \mathrm{~h}$ (i.e., $C_{\mathrm{DL}, 0}$ and $\mathrm{R}_{\mathrm{CT}, 0)}$. In this way, discrepancies in the absolute values are eliminated, allowing for better graph visibility. Figure 9 shows that all curves present an initial transient in which $C_{\mathrm{DL}}$ increases and $R_{\text {Ст }}$ decreases rapidly. This is caused by the culture medium's adaptation to a change in external conditions, when Petri dish passes from being inoculated at ambient temperatures to being put in the thermostatic chamber at $35^{\circ} \mathrm{C}$. Then, the curves referring to the non-inoculated plate are characterised only by irrelevant variations after the initial transient, which are mostly due to medium partial drying. In fact, we always noticed water droplets on plate covers at the end of each test, because the plastic bag containing the plate does not allow air exchange with the outside. On the other hand, the curves related to the inoculated Petri dish show the typical phases of a bacterial growth curve [6]:

- a lag phase, in which $C_{\mathrm{DL}}$ and $\mathrm{R}_{\mathrm{CT}}$ are almost constant as the bacteria are adapting themselves to the surrounding environmental conditions;
- a $\log$ phase, in which $C_{\mathrm{DL}}$ increases and $\mathrm{R}_{\mathrm{CT}}$ decreases, since the bacteria multiply and reach a number that is large enough to be detected. This is in agreement with what is reported in the literature, since one of the by-products of bacterial metabolism is an increase of ion concentration in the medium, which modifies the electrochemical properties of the electrode/medium interface [6];

- a stationary phase (barely visible in Figure 9), characterised by a lack of variations in $C_{\mathrm{DL}}$ or $R_{\mathrm{CT}}$, because of the equilibrium between live and dead bacteria present in the culture medium; and

- a death phase, in which $C_{\mathrm{DL}}$ decreases and $R_{\mathrm{CT}}$ increases, because a growing number of bacteria die, since there is no more medium available for all.

The most interesting tract of these curves is the one comprising the lag and log phases, as the evident change in the curve slope observed between them identifies when the system is able to detect bacterial growth. Figure 9 highlights that the detection time is equal to four hours given the considered initial concentration.

Figure 10 shows the $R_{M}$ time behaviour, referring to the Petri dishes inoculated with $4.5 \cdot 10^{8} \mathrm{CFU} / \mathrm{ml}$ (Figure 10a), 4.5.107 $\mathrm{CFU} / \mathrm{ml}$ (Figure 10b), and $4.5 \cdot 10^{6} \mathrm{CFU} / \mathrm{ml}$ (Figure 10c), together with the curves related to the non-inoculated plates. As in Figure 9 for $C_{\mathrm{DL}}$ and $R_{\mathrm{CT}}, R_{\mathrm{M}}$ is normalised with respect to its value $R_{\mathrm{M}, 0}$, assumed at time $=0 \mathrm{~h}$. The reported curves have an initial transient caused by the medium settling to the
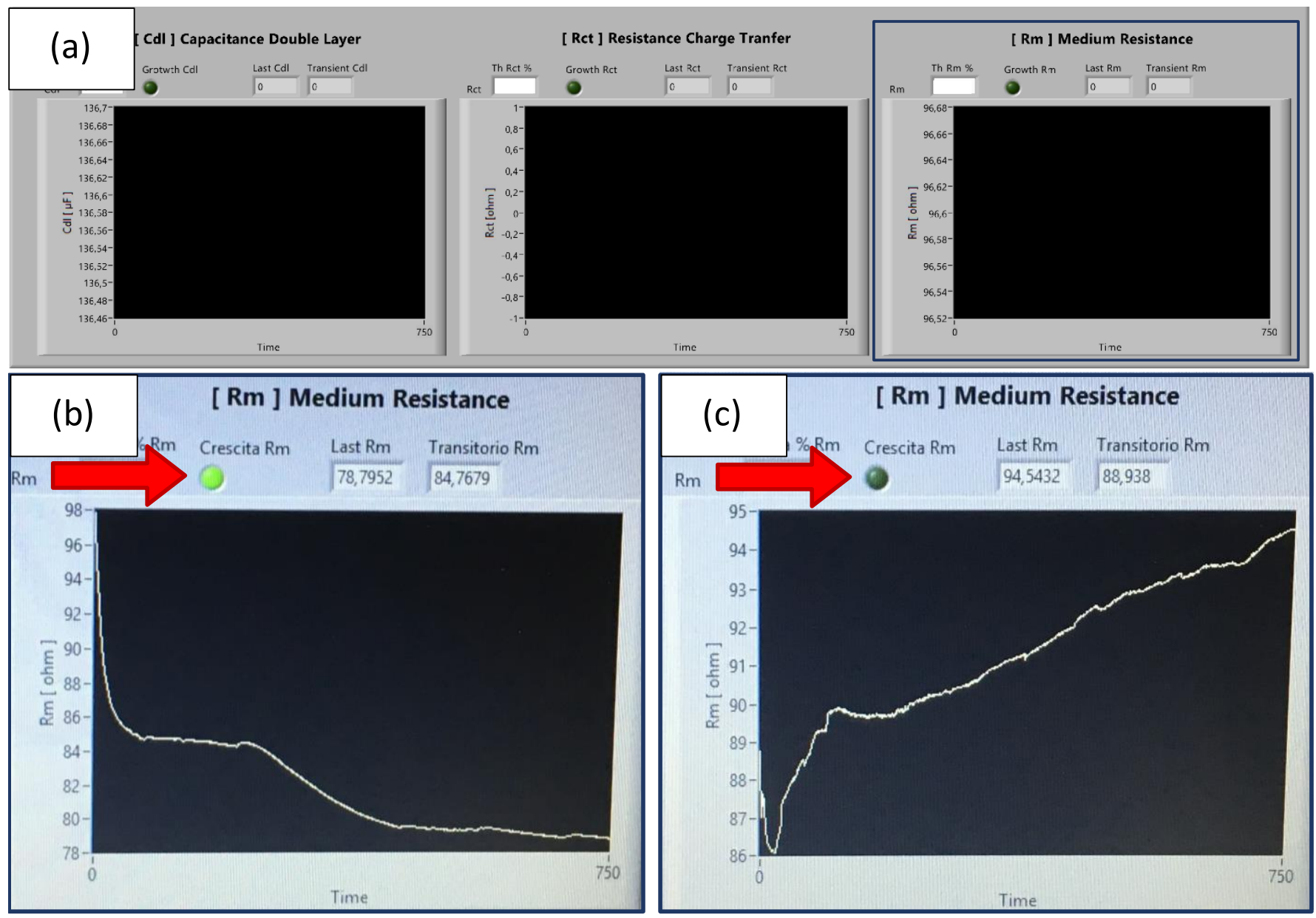

Figure 11. VI user interface reporting $R_{\mathrm{M}}$ real-time information during a test: (a) a global view for monitoring all parameters; (b) a case related to an inoculated Petri dish; and (c) a case related to a non-inoculated Petri dish. 
environmental conditions. Afterwards, those related to the inoculated plates present a decreasing $\log$ phase until the end of the test (in fact, $R_{M}$ has slower dynamics than $C_{\mathrm{DL}}$ and $R_{\mathrm{CT}}$ ). Furthermore, the behaviour of $R_{M}$ supports what is stated in the literature: Ion release through bacterial metabolism contributes to an increase of medium conductance [6]. On the contrary, the curves referring to the non-inoculated Petri dishes maintain a constant trend (it may even be increasing in the case that the medium is drying as the conductance falls). In addition, Figure 10 evidences that the $\log$ phase begins later; therefore, the system detection time augments when $C_{0}$ decreases, as expected.

The results of the preliminary tests demonstrate that the measuring system is able to automatically detect bacterial growth in Petri dishes and discriminate between plates inoculated with different pathogen concentrations. In addition, it can monitor growth continuously over time, during its various phases.

\subsection{Tests with the WASPLab ${ }^{\circledR}$}

This section especially focuses on system interaction with the user and other devices in order to better highlight its operation in an Industry 4.0 framework.

Figure 11 shows the VI user interface while the system is working during a test. Figure 11a provides a global view of the interface, allowing for the monitoring of all parameters. Figure $11 \mathrm{~b}$ and Figure 11c focus on real-time information concerning $R_{\mathrm{M}}$ related to an inoculated and a non-inoculated Petri dish, respectively. In particular, besides the growth curve reported in the graph, the 'Last $R m$ ' indicator refers to the last $R_{M}$ value obtained when the pictures were taken, while 'Transitorio Rm' is the reference that is considered for comparison with the last value in order to recognise whether bacterial growth has occurred. Furthermore, in Figure 11b, 'Crescita Rm' LED (indicated by an arrow) is on, which means that the system generated an alarm due to its detection of bacterial growth in the analysed Petri dish. As a proof, the displayed curve is similar to those shown in Figure 10 for the inoculated plates. On the contrary, at the same time, LED in Figure 11c is off, and the reported curve has an increasing trend. This proves that the system successfully indicated that the examined plate had not been inoculated.

Table 1 reports some data extracted from a test in which $C_{0}$ $=4 \cdot 5 \cdot 10^{7} \mathrm{CFU} / \mathrm{ml}$. The first column on the left shows the time in hours and minutes from the test's commencement. The second, fourth, and sixth columns contain the $C_{\mathrm{DL}}, R_{\mathrm{CT}}$, and $R_{\mathrm{M}}$ values, respectively, related to an inoculated Petri dish. Finally, the third, fifth, and seventh columns include the parameter percentage variations with respect to their corresponding values at $1 \mathrm{~h}$ i.e. when it is reasonable to consider the initial transient as terminated. In this way, such variation is calculated starting from the lag phase (reference). For space reasons, Table 1 shows only the data obtained during the sixth hour of the examined test i.e. the interval including the detection time. As explained in Section 2.3 , the system exerts its monitoring function by comparing the parameter variations with proper thresholds. Based on statistical

Table 1. Data on the model parameters for an inoculated Petri dish obtained during the sixth hour of a test in which $C_{0}=4 \cdot 5 \cdot 10^{7} \mathrm{CFU} / \mathrm{ml}$.

\begin{tabular}{|c|c|c|c|c|c|c|}
\hline Time & $C_{\mathrm{DL}}(\mu \mathrm{F})$ & $\%$ var, $C_{\mathrm{DL}}$ & $R_{\mathrm{CT}}(\Omega)$ & $\%$ var, $R_{c t}$ & $R_{\mathrm{M}}(\Omega)$ & $\%$ var, $R_{\mathrm{M}}$ \\
\hline $5 \mathrm{~h} 00 \mathrm{~min}$ & 195.20 & 2.04 & 22.23 & -1.90 & 74.70 & -1.75 \\
\hline $5 \mathrm{~h} 02 \mathrm{~min}$ & 195.98 & 2.44 & 22.23 & -1.91 & 74.72 & -1.73 \\
\hline $5 \mathrm{~h} 04 \mathrm{~min}$ & 196.04 & 2.47 & 22.22 & -1.94 & 74.71 & -1.74 \\
\hline $5 \mathrm{~h} 06 \mathrm{~min}$ & 195.85 & 2.38 & 22.21 & -2.00 & 74.70 & -1.76 \\
\hline $5 \mathrm{~h} 08 \mathrm{~min}$ & 196.08 & 2.50 & 22.19 & -2.07 & 74.69 & -1.76 \\
\hline $5 \mathrm{~h} 10 \mathrm{~min}$ & 196.11 & 2.51 & 22.18 & -2.11 & 74.68 & -1.78 \\
\hline $5 \mathrm{~h} 12 \mathrm{~min}$ & 196.32 & 2.62 & 22.21 & -1.98 & 74.68 & -1.78 \\
\hline $5 \mathrm{~h} 14 \mathrm{~min}$ & 196.17 & 2.54 & 22.20 & -2.06 & 74.66 & -1.81 \\
\hline $5 \mathrm{~h} 16 \mathrm{~min}$ & 196.32 & 2.62 & 22.20 & -2.04 & 74.65 & -1.82 \\
\hline $5 \mathrm{~h} 18 \mathrm{~min}$ & 196.65 & 2.80 & 22.18 & -2.14 & 74.65 & -1.82 \\
\hline $5 \mathrm{~h} 20 \mathrm{~min}$ & 196.32 & 2.63 & 22.11 & -2.42 & 74.61 & -1.88 \\
\hline $5 \mathrm{~h} 22 \mathrm{~min}$ & 196.95 & 2.96 & 22.11 & -2.45 & 74.61 & -1.88 \\
\hline $5 \mathrm{~h} 24 \mathrm{~min}$ & 197.51 & 3.25 & 22.08 & -2.58 & 74.61 & -1.88 \\
\hline $5 \mathrm{~h} 28 \mathrm{~min}$ & 197.12 & 3.04 & 22.04 & -2.76 & 74.56 & -1.94 \\
\hline $5 \mathrm{~h} 30 \mathrm{~min}$ & 197.23 & 3.10 & 22.00 & -2.91 & 74.55 & -1.95 \\
\hline $5 \mathrm{~h} 32 \mathrm{~min}$ & 197.84 & 3.42 & 22.02 & -2.85 & 74.56 & -1.94 \\
\hline $5 \mathrm{~h} 34 \mathrm{~min}$ & 197.65 & 3.32 & 21.96 & -3.11 & 74.53 & -1.98 \\
\hline $5 \mathrm{~h} 36 \mathrm{~min}$ & 197.77 & 3.38 & 21.96 & -3.10 & 74.52 & -1.99 \\
\hline $5 \mathrm{~h} 38 \mathrm{~min}$ & 198.58 & 3.81 & 21.98 & -3.01 & 74.54 & -1.96 \\
\hline $5 \mathrm{~h} 40 \mathrm{~min}$ & 198.77 & 3.91 & 21.95 & -3.15 & 74.53 & -1.98 \\
\hline $5 \mathrm{~h} 42 \mathrm{~min}$ & 198.38 & 3.70 & 21.90 & -3.38 & 74.49 & -2.03 \\
\hline $5 \mathrm{~h} 44 \mathrm{~min}$ & 198.93 & 3.99 & 21.89 & -3.41 & 74.50 & -2.02 \\
\hline $5 \mathrm{~h} 46 \mathrm{~min}$ & 199.40 & 4.23 & 21.85 & -3.58 & 74.49 & -2.03 \\
\hline $5 \mathrm{~h} 48 \mathrm{~min}$ & 199.59 & 4.33 & 21.80 & -3.82 & 74.47 & -2.05 \\
\hline $5 \mathrm{~h} 50 \mathrm{~min}$ & 200.28 & 4.70 & 21.78 & -3.91 & 74.47 & -2.05 \\
\hline $5 \mathrm{~h} 52 \mathrm{~min}$ & 200.62 & 4.87 & 21.75 & -4.02 & 74.47 & -2.06 \\
\hline $5 \mathrm{~h} 58 \mathrm{~min}$ & 200.67 & 4.90 & 21.64 & -4.50 & 74.53 & -1.97 \\
\hline $6 \mathrm{~h} 00 \mathrm{~min}$ & 201.57 & 5.37 & 21.65 & -4.48 & 74.60 & -1.89 \\
\hline
\end{tabular}


$\leftarrow$ Rispondi $\ll$ Rispondi a tutti $\rightarrow$ Inoltra $\ldots$

\section{bacterial growth detection}

system.adaptive <system.adaptive@gmail.com>

\section{A: Michele Bona}

Dear user,

this is to alert you that measuring system detected bacterial growth in a Petri dish.

Please check the data and remove the Petri dish from WASPLab incubator.

Thank you.

Figure 12. Email message sent by the system to a specified address when it detects bacterial growth.

considerations on the obtained data, we set these thresholds to $2.50 \%,-2.00 \%$, and $-2.00 \%$ for $C_{\mathrm{DL}}, R_{\mathrm{CT}}$, and $R_{\mathrm{M}}$, respectively. Such values represent a good trade-off. In fact, increasing them would postpone the detection time too much, while decreasing them would increase the probability of false positives, since the system would generate an alarm even if the bacterial growth did not occur. Considering the data of Table 1 in depth, it may be observed that $C_{\mathrm{DL}}$ is the first parameter that exceeds its threshold, after 5 hours and 18 minutes. We activated a control, ensuring that the threshold was consecutively overcome for 10 minutes in a continuative way. This prevents the signal noise from leading to wrong conclusions. Then, $R_{C T}$ did the same after 5 hours and 24 minutes. Finally, the $R_{M}$ threshold was exceeded after 5 hours and 52 minutes. At this point, the system generated the alarm, as it detected bacterial growth. Waiting for all parameters to overcome their own thresholds increases the reliability of the results. In fact, $C_{\mathrm{DL}}$ and $R_{\mathrm{CT}}$ provide a quicker response than $R_{M}$, but the latter is more stable over time. For this reason, the system records the events regarding $C_{\mathrm{DL}}$ and $\mathrm{R}_{\mathrm{CT}}$, but it alerts the user about bacterial growth detection only once the data on $R_{\mathrm{M}}$ reports it.

Figure 12 shows the email message that is sent automatically by the system (from a Gmail account created ad hoc) to a specified address (in this case, the corresponding author's), when it detects bacterial growth in a Petri dish. Such message is simply a notification, which invites the recipient to check real-time data (which is shared on the cloud) and to extract the Petri dish of interest from the WASPLab $®$ incubator for further analysis.

In general, these achievements show how the developed measuring system can continuously monitor bacterial growth. Furthermore, the connectivity between the system, the

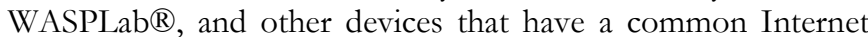
connection (e.g., PCs, tablets, smartphones) allows a user to be informed about the analyses that are in progress and to act on the system at any time and from remote locations. In fact, all data about the monitored parameters and information on the system operating conditions is shared on the cloud, and authorised devices can access the system remotely.

\section{CONCLUSION}

This article has presented a measuring system that allows for the automatic detection of bacterial growth in Petri dishes, while they are inside the incubators of the WASPLab ${ }^{\circledR}$ platform (COPAN Italia S.p.A.). System operation relies on real-time monitoring of three parameters derived from the impedance measurement related to analysed Petri dishes. Furthermore, the system can be easily reconfigured according to user requirements. Finally, it presents features that enable its connectivity with the WASPLab ${ }^{\circledR}$ and other devices, creating a structure that is compliant with the Industry 4.0 model and applicable to the microbiology field. We undertook different tests aiming to evaluate system detection abilities. In particular, we explained how the system is prepared for the tests. We provided a description of the experimental setups and test protocols used. We then presented the obtained results.

This work's achievements open the possibility for a collaboration between the proposed system and the WASPLab ${ }^{\circledR}$ or an integration of the former with the latter, implementing the model of a Smart Factory. This could lead to many potential benefits. In fact, the generation of quantitative data about bacterial growth from continuous impedance measurements and precocious growth detection in incubated Petri dishes, besides and independent from image acquisition by the WASPLab ${ }$, could further enhance platform analysis performances and reduce time wastage. Then, the huge amount of generated data can be exchanged between the system and other devices, permitting more effective supervision by users who are in remote locations, too.

\section{ACKNOWLEDGEMENT}

The authors would like to thank Roberto Paroni and Giorgio Triva from COPAN Italia S.p.A. for their support during the different phases of the research activity.

\section{REFERENCES}

[1] World Health Organization, World Health Statistics 2018: Monitoring Health for the SDGs, Sustainable Development Goals, Geneva, Switzerland, World Health Organization, 2018, ISBN 978-92-4-156558-5.

[2] World Health Organization, Global Antimicrobial Resistance Surveillance System (GLASS) Report: Early Implementation 2016-2017, Geneva, Switzerland, World Health Organization, 2018, ISBN 978-92-4-151344-9.

[3] D.Ivnitski, I.Abdel-Hamid, P.Atanasov, E.Wilkins, Biosensors for detection of pathogenic bacteria, Biosens. Bioelectron., 14 (1999) pp. 599-624.

[4] M.Rubab, H.Muhammad Shahbaz, A.N.Olaimat, D.-H.Oh, Biosensors for rapid and sensitive detection of Staphylococcus aureus in food, Biosens. Bioelectron., 105 (2018) pp. 49-57.

[5] H.E.Giana, L.Silveira Jr., R.A.Zângaro, M.T.T.Pacheco, Rapid identification of bacterial species by fluorescence spectroscopy and classification through principal components analysis, J. Fluoresc., 13 (2003) pp. 489-493.

[6] L.Yang, R.Bashir, Electrical/electrochemical impedance for rapid detection of foodborne pathogenic bacteria, Biotechnol. Adv., 26 (2008) pp. 135-150.

[7] H.Baccar, M.B.Mejri, I.Hafaiedh, T.Ktari, M.Aouni, A.Abdelghani, Surface plasmon resonance immunosensor for bacteria detection, Talanta, 82 (2010) pp. 810-814.

[8] O.Braissant, D.Wirz, B.Göpfert, A.U.Daniels, Use of isothermal microcalorimetry to monitor microbial activities, FEMS Microbiol. Lett., 303 (2010) pp. 1-8. 
[9] I.O.L.McHugh, A.Ling Tucker, Flow cytometry for the rapid detection of bacteria in cell culture production medium, Cytometry A, 71 (2007) pp. 1019-1026.

[10] O.Dauwalder, L.Landrieve, F.Laurent, M.de Montclos, F.Vandenesch, G.Lina, Does bacteriology laboratory automation reduce time to results and increase quality management? Clin. Microbiol. Infect., 22 (2016) pp. 236-243.

[11] A. S. Johnson, C. Touchie, D. J. M. Haldane, K. R. Forward, Fourday incubation for detection of bacteremia using the BACTEC 9240, Diagn. Microbiol. Infect. Dis., 38, (2000), pp. 195-199.

[12] M. Mueller-Premru, S. Jeverica, L. Papst, E. Nagy, Performance of two blood culture systems to detect anaerobic bacteria. Is there any difference?, Anaerobe, 45, (2017), pp. 59-64.

[13] H.Totty, M.Ullery, J.Spontak, J.Viray, M.Adamik, B.Katzin, W.M.Dunne, P.Deol, A controlled comparison of the BacT/ALERT ${ }^{\circledR}$ 3D and VIRTUOTM microbial detection systems, Eur. J. Clin. Microbiol. Infect. Dis. 36(10) (2017) pp. 1795-1800.

[14] M.R.Jacobs, T.Mazzulli, K.C.Hazen, C.E.Good, A.M.Abdelhamed, P.Lo, B.Shum, K.P.Roman, D.C.Robinson, Multicenter clinical evaluation of BacT/Alert Virtuo blood culture system, J. Clin. Microbiol., 55(8) (2017) pp. 2413-2421.

[15] G.Zsivanovits, F.Szigeti, C.Mohacsi-Farkas, 'Investigation of antimicrobial inhibition effect of quince fruit extract by rapid impedance method', Proc. of International Scientific-Practical Conference on Food, Technologies and Health, 2013, Plovdiv, Bulgaria, pp. 264-270.

[16] P.Fernández, J.A.Gabaldón, M.J.Periago, Detection and quantification of Alicyclobacillus acidoterrestris by electrical impedance in apple juice, Food. Microbiol. 68 (2017) pp. 34-40.

[17] P.M.Fratamico, T.P.Strobaugh, M.B.Medina, A.G.Gehring, Detection of Escherichia coli O157:H7 using a surface plasmon resonance biosensor, Biotechnol. Tech., 12(7) (1998) pp. 571-576.

[18] C.Quiblier, M.Jetter, M.Rominski, F.Mouttet, E.C.Böttger, P.M.Keller, M.Hombach, Performance of Copan WASP for routine urine microbiology, J. Clin. Microbiol., 54 (2016) pp. 585592.

[19] The WASPLab ${ }^{\circledR}$ platform [Online] Available: http://products.copangroup.com/index.php/products/labautomation/wasplab
[20] P.P.Bourbeau, N.A.Ledeboer, Automation in clinical microbiology, J. Clin. Microbiol., 51 (2013) pp. 1658-1665.

[21] V.Villani, L.Sabattini, J.N.Czerniaki, A.Mertens, B.Vogel-Heuser, C.Fantuzzi, 'Towards modern inclusive factories: a methodology for the development of smart adaptive human-machine interfaces', Proc. of the 22nd IEEE International Conference on Emerging Technologies and Factory Automation (ETFA), 12-15 Sept., 2017, Limassol, Cyprus, pp. 1-7.

[22] M.Hermann, T.Pentek, B.Otto, 'Design principles for Industrie 4.0 scenarios', Proc. of the $49^{\text {th }}$ Hawaii International Conference on Systems Sciences (HICSS), 5-8 Jan, 2016, Koloa, Hawaii, USA, pp. 3928-3937.

[23] P.Samaranayake, K.Ramanathan, T.Laosirihongthong, Implementing industry $4.0 \quad-$ a technological readiness perspective', Proc. of the 2017 IEEE International Conference on Industrial Engineering and Engineering Management (IEEM), 1013 Dec., 2017, Singapore, pp. 529-533.

[24] M.Bona, P.Bellitti, E.Sardini, M.Serpelloni, 'Study for the integration of a measuring system to an automated platform for monitoring the growth of bacterial cultures', Proc. of the $1^{\text {st }}$ IEEE International Workshop on Metrology for Industry 4.0 and IoT, 16-18 Apr., 2018, Brescia, Italy, pp. 266-270.

[25] M.Bona, M.Borghetti, P.Bellitti, M.Serpelloni, E.Sardini, 'A concept sensor-based system to be integrated in an existing automated platform monitoring bacterial growth', Proc. of the $23^{\text {rd }}$ International Conference on Engineering, Technology and Innovation (ICE/IEEE ITMC), 27-29 Jun., 2017, Funchal, Madeira Island, Portugal, pp. 490-497.

[26] P. Bellitti, M. Bona, M. Borghetti, E. Sardini, M. Serpelloni, Flexible monitoring system for automated detection of bacterial growth in a commercial specimen processing platform, Proc. of $3^{\text {rd }}$ IEEE International Forum on Research and Technologies for Society and Industry (RTSI), Modena, Italy, Sept. 11-13, 2017, pp. 207-212.

[27] J.E.B.Randles, Kinetics of rapid electrode reactions, Discuss. Faraday Soc., 1 (1947) pp. 11-19.

[28] AD5933 datasheet [Online] Available: http://www.analog.com/media/en/technicaldocumentation/data-sheets/AD5933.pdf 